\title{
The initial study of EBNA-2 polymorphisms in nasopharyngeal carcinoma in Vietnam
}

Ngo Dong Kha ${ }^{1}$, Huynh Thị Mong Tuyen ${ }^{2}$, Lam Hong Ngoc ${ }^{2}$, Thieu Hong Hue Le ${ }^{2}$, Quang Anh Tuan², Lao Duc Thuan ${ }^{2}$, Le Huyen Ai Thuy ${ }^{2 *}$

${ }^{1}$ University of Science, Vietnam National University Ho Chi Minh City, Vietnam

${ }^{2}$ Ho Chi Minh City Open University, Vietnam

*Corresponding author: thuy.lha@ ou.edu.vn

\begin{tabular}{|c|c|}
\hline ARTICLE INFO & ABSTRACT \\
\hline $\begin{array}{l}\text { DOI:10.46223/HCMCOUJS. } \\
\text { tech.en.8.1.337.2018 }\end{array}$ & $\begin{array}{l}\text { Epstein-Barr virus (EBV) infection is the main cause of } \\
\text { Nasopharyngeal Carcinoma (NPC). EBNA-2, one of the most } \\
\text { important genes participating in the formation of NPC, also helps } \\
\text { EBV evade an attack on the immune system. EBNA-2 has } 4 \\
\text { variants including E2-A, E2-B, E2-C and E-2D, of which E2-A } \\
\text { and E2-C are the characterized variants for NPC. This study aimed }\end{array}$ \\
\hline Received: February $06^{\text {th }}, 2018$ & to evaluate the variations of $E B N A-2$ in NPC biopsy samples of \\
\hline Revised: March $14^{\text {th }}, 2018$ & Vietnamese patients. This initial study used 10 biopsy samples, \\
\hline Accepted: April $16^{\text {th }}, 2018$ & $\begin{array}{l}\text { which were positively confirmed to NPC, collected from Cho Ray } \\
\text { Hospital. Nested PCR - nucleotide sequencing was applied to } \\
\text { analyze the variants of EBNA-2. The results showed that } 8 \text { out of } \\
10 \text { samples, accounting for } 80 \% \text {, were positive for } E B N A-2 \text {. } \\
\text { Additionally, only two variants, E- } 2 \text { A and E-2C were detected in }\end{array}$ \\
\hline Ceywords: & our study, in which, E2-A subtype was identified as the \\
\hline $\begin{array}{l}\text { E-2A, E-2C, EBNA-2, } \\
\text { nasopharyngeal carcinoma, } \\
\text { SNPs }\end{array}$ & $\begin{array}{l}\text { predominant subtype. These findings would provide initial data } \\
\text { about the potential contribution of EBNA-2 polymorphisms to the } \\
\text { etiology of NPC in the Vietnamese population. }\end{array}$ \\
\hline
\end{tabular}

\section{Introduction}

Nasopharyngeal carcinoma (NPC) is a human malignant disease derived from epithelial cells. There is a striking, geographic and ethnic distribution of this disease which is common in Southern China, Southern Asia (da Costa, Marques-Silva, \& Moreli, 2015; Dai, Zheng, Cheung, \& Lung, 2016; McDermott, Dutt, \& Watkinson, 2001; Pathmanathan, Prasad, Sadler, Flynn, \& Raab-Traub, 1995). According to Globocan, NPC is considered one of the five most common cancers in men in Vietnam in 2012, of which the number of infected cases is 3,301 (ASR = $7.7 / 100,000$ cases) and of mortality cases is 1,931 (ASR $=4.8 / 100,000$ cases). Besides, there were 1,630 infected cases $(\mathrm{ASR}=3.4 / 100,000$ cases) and 954 mortality cases (ASR = $2 / 100,000$ cases) in women. Previous studies showed that major etiological factors proposed for NPC pathogenesis have been significant to the EBV infection (Lo, To, \& Huang, 2004; Tsao et al., 2014; Yang et al., 2005). EBV, also known as human herpesvirus 4, a member of the Herpesviridae family, has been proven to be significantly associated with many human cancers 
such as Burkitt's lymphoma, gastric carcinoma, nasopharyngeal carcinoma, and so on (Lo et al., 2004; Tsao et al., 2014). Understanding the role of EBV latent genes is essential for identifying the mechanism underlying EBV-induced cell transformation and immune evasion (Ko, 2015). Among them, EBNA-2 has been shown to be essential for the infection of EBV and existed in the cellular. Numerous studies have long been trying to identify NPC-specific EBV subtypes based on the sequence variation of $E B N A-2$ to display a characteristic geographical prevalence and distribution. The EBNA-2 polymorphism was classified into four subtypes including E2-A, E2-B, E2-C and E2-D. To the best of our knowledge, there has been no research on the classification of the EBNA-2 subtype in the Vietnamese population. This study, therefore, aims to analyze NPC biopsy samples collected from Vietnamese NPC patients for the first time to examine whether there is a remarkable association between certain EBNA-2 subtypes and NPC from a distinct geographical location.

\section{Materials and method}

\subsection{Ethics statement}

Institutional Ethics Board approval was obtained from the Medical Ethics Committee of the Cho Ray Hospital, Ho Chi Minh City, Vietnam. The permit from the Ethical committee was under decision number 516/BVCR-HDDD, Cho Ray Hospital, Ho Chi Minh City, Vietnam. All the samples used in this study were agreed by Cho Ray Hospital and obtained from all participants in this clinical trial.

\subsection{Sample collection}

10 NPC tumor biopsies were collected with informed consent from NPC patients at Cho Ray hospital. All samples were submitted to the histopathological department and then, proved histologically to have NPC by hematoxylin and eosin examination. Total genomic DNA extraction was isolated from biopsy samples by using Phenol/chloroform method. The samples were lysed in lysis buffer (10 mM Tris- $\mathrm{HCl} \mathrm{pH} \mathrm{=8,} 10 \mathrm{mM}$ EDTA, $150 \mathrm{mM} \mathrm{NaCl}, 2 \%$ SDS) containing Proteinase $\mathrm{K}(0.1 \mathrm{mg} / \mathrm{ml})$. Then, total genomic DNA extraction was isolated and purified by using standard phenol-chloroform and ethanol precipitation. The quality and purity of DNA extraction were measured by the evaluation of the $\mathrm{A}_{260} / \mathrm{A}_{280}$ proportion. Then, the DNA solution was the store at EDTA $0.5 \mathrm{M},-20^{\circ} \mathrm{C}$ for PCR assay.

\subsection{Nested - Polymerase Chain Reaction assay (Nested - PCR)}

Nested - PCR and direct sequencing were used to detect the sequence of EBNA-2. The primers of stage 1 (outer primer) and stage 2 (internal primer) were shown in Table 1. Total volume reaction is $15 \mu \mathrm{l}$ containing $2 \mu \mathrm{l}$ DNA isolation (for stage 1) or $2 \mu \mathrm{l} \mathrm{PCR}$ product of stage 1 (for stage 2), 0.75 unit iTaq DNA polymerase, $0.5 \mu 1$ each primer, $7.5 \mu 1$ MyTaqTM Mix and water up to $15 \mu$. Thermal cycling was set up at $95^{\circ} \mathrm{C}$ for 5 minutes, followed by 35 cycles of denaturation at $95^{\circ} \mathrm{C}$ for 30 seconds, annealing at $55^{\circ} \mathrm{C}$ for 30 seconds, extension at $72^{\circ} \mathrm{C}$ for 30 seconds, and a final extension at $72^{\circ} \mathrm{C}$ for $10 \mathrm{~min}$. Finally, The PCR products were expressed by electrophoresis on $2 \%$ agarose gel and DNA was dyed with ethidium bromide. 


\section{Table 1}

The primer sequences used in this study

\begin{tabular}{|c|c|c|c|c|}
\hline Region & Stage & Primer & Sequence (3' - 5') & Ref \\
\hline \multirow{4}{*}{$\begin{array}{l}\text { EBNA2-A } \\
(163-294)\end{array}$} & \multirow{2}{*}{1} & $\mathrm{E} 2 \mathrm{~A}-\mathrm{OF}$ & GCTATGCGAATGCTTTGG & \multirow{8}{*}{$\begin{array}{l}\text { (Wang et } \\
\text { al., 2012) }\end{array}$} \\
\hline & & E2A-OR & GAGTCTTAGAGGGTTGCG & \\
\hline & \multirow{2}{*}{2} & E2A-IF & CTATGCGAATGCTTTGGA & \\
\hline & & E2A-IR & TTGTTGGTCGTTGATGAC & \\
\hline \multirow{4}{*}{$\begin{array}{l}\text { EBNA2-C } \\
(357-486)\end{array}$} & \multirow{2}{*}{1} & E2C-OF & AGAACCACGGTCCCCGACTGTA & \\
\hline & & E2C-OR & TGCTGAGAGCAAGGCACCAATT & \\
\hline & \multirow{2}{*}{2} & E2C-IF & ACGGTCCCCGACTGTATTTTAT & \\
\hline & & E2C-IR & TTTTGGCAAGCCTTCCTT & \\
\hline
\end{tabular}

Source: The researcher's data analysis

\section{Results and discussion}

\subsection{Sequence variation data of EBNA-2 in NPC biopsy samples}

In this study, nested-PCR was used to amplify the target sequence. As a result, 8 out of 10 samples, accounting for $80 \%$, were positive for $E B N A-2$, which yielded a PCR product of 551 bps-band and 788 bps-band as shown in Figure 1. The amplification of EBNA-2 fragments, including $E B N A-2 A$ and $E B N A-2 C$, was determined by Sanger sequencing as shown in Figure 2.

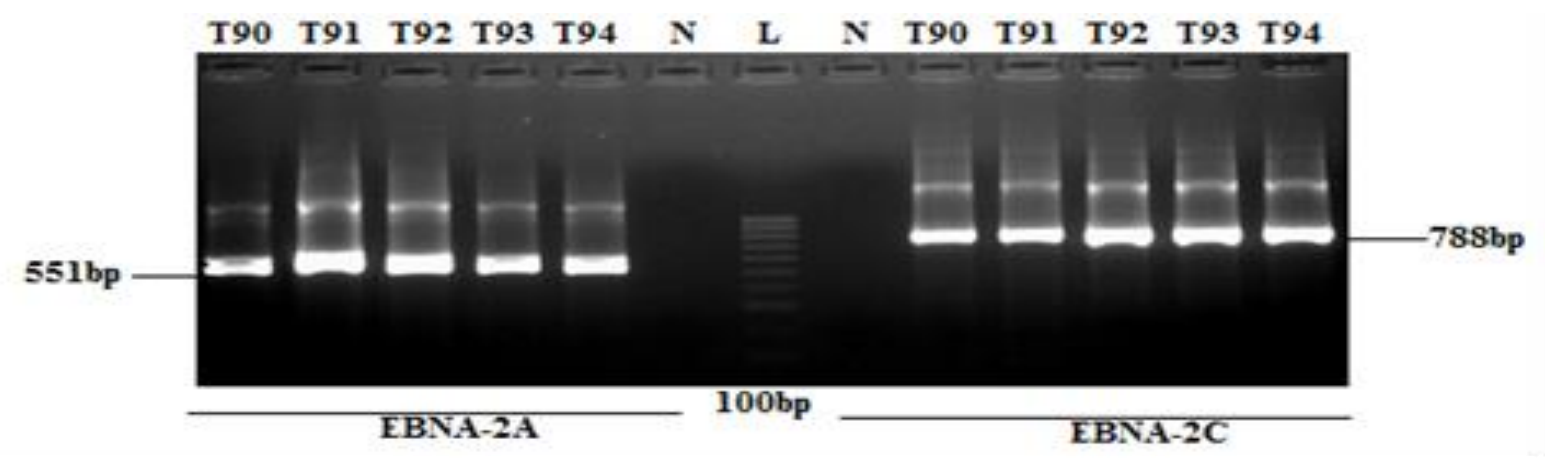

Figure 1. Agarose gel electrophoresis of the PCR products of representative NPC biopsy samples. N: negative control. Ladder: 100 bps 


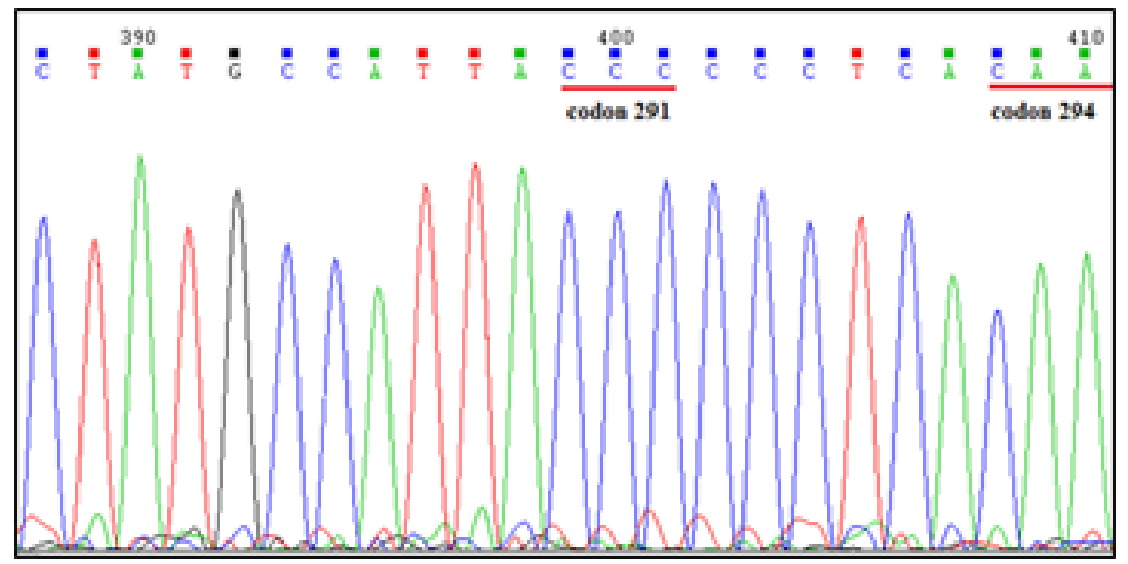

Figure 2. Sequence determination of $E B N A-2 A$ of representative NPC biopsy samples by Sanger sequencing

Table 2

Variant positions of EBNA-2

\begin{tabular}{|c|c|c|c|c|c|c|c|c|c|}
\hline \multirow{3}{*}{$\begin{array}{l}\text { Sub } \\
\text { types }\end{array}$} & \multirow{2}{*}{$\begin{array}{c}\text { Amino } \\
\text { Acid }\end{array}$} & 151 & 160 & 163 & 165 & 185 & 195 & $200 \rightarrow 217$ & 201 \\
\hline & & \multicolumn{8}{|c|}{ Self-association domain } \\
\hline & WT & $\begin{array}{c}\text { ATT } \\
\text { Ile } \\
\end{array}$ & $\begin{array}{l}\text { CCT } \\
\text { Pro } \\
\end{array}$ & $\begin{array}{c}\text { AGG } \\
\text { Arg } \\
\end{array}$ & $\begin{array}{c}\text { GTC } \\
\text { Val } \\
\end{array}$ & $\begin{array}{l}\text { CAA } \\
\text { Gln } \\
\end{array}$ & $\begin{array}{r}\text { ATG } \\
\text { Met }\end{array}$ & $\begin{array}{c}51 \text { nucleotide } \\
18 \text { a.a } \\
\end{array}$ & $\begin{array}{l}\text { ACC } \\
\text { Thr } \\
\end{array}$ \\
\hline \multirow{6}{*}{ E2-A } & $\mathrm{T} 79$ & $\begin{array}{l}--\mathrm{G} \\
\text { Met }\end{array}$ & $\begin{array}{c}--\mathrm{C} \\
+\end{array}$ & $\begin{array}{l}\text { GT- } \\
\text { Val }\end{array}$ & $\begin{array}{c}-\mathrm{A} \\
+\end{array}$ & $\begin{array}{l}-\mathrm{G}- \\
\text { Arg }\end{array}$ & $\begin{array}{l}-\mathrm{C}- \\
\text { Thr }\end{array}$ & $\begin{array}{c}--- \\
*\end{array}$ & $\begin{array}{l}--\mathrm{T} \\
+\end{array}$ \\
\hline & T80 & $\begin{array}{l}--\mathrm{G} \\
\text { Met }\end{array}$ & $\begin{array}{c}-\mathrm{C} \\
+ \\
\end{array}$ & $\begin{array}{l}\text { GT- } \\
\text { Val }\end{array}$ & $\begin{array}{c}-\mathrm{A} \\
+\end{array}$ & $\begin{array}{l}-\mathrm{G}- \\
\text { Arg }\end{array}$ & --- & Del & *-- \\
\hline & T86 & $\begin{array}{l}--\mathrm{G} \\
\text { Met }\end{array}$ & $\begin{array}{l}--\mathrm{C} \\
+ \\
\end{array}$ & $\begin{array}{l}\text { GT- } \\
\text { Val }\end{array}$ & $\begin{array}{c}-\mathrm{A} \\
+ \\
\end{array}$ & $\begin{array}{l}-\mathrm{G}- \\
\text { Arg }\end{array}$ & $\begin{array}{l}-\mathrm{C}- \\
\text { Thr }\end{array}$ & --- & $\begin{array}{l}--\mathrm{T} \\
+ \\
\end{array}$ \\
\hline & T90 & $\begin{array}{l}--G \\
\text { Met }\end{array}$ & *- & $\begin{array}{l}\text { GT- } \\
\text { Val }\end{array}$ & $\begin{array}{c}-\mathrm{A} \\
+ \\
\end{array}$ & $\begin{array}{l}-\mathrm{G}- \\
\text { Arg }\end{array}$ & --- & Del & *-- \\
\hline & T91 & $\begin{array}{l}--\mathrm{G} \\
\text { Met }\end{array}$ & $\begin{array}{l}--\mathrm{C} \\
+ \\
\end{array}$ & $\begin{array}{l}\text { GT- } \\
\text { Val }\end{array}$ & $\begin{array}{c}-\mathrm{A} \\
+ \\
\end{array}$ & $\begin{array}{l}-\mathrm{G}- \\
\text { Arg }\end{array}$ & $\begin{array}{l}-\mathrm{C}- \\
\text { Thr }\end{array}$ & *-- & $\begin{array}{l}--\mathrm{T} \\
+ \\
\end{array}$ \\
\hline & T97 & $\begin{array}{l}--G \\
\text { Met }\end{array}$ & --- & $\begin{array}{l}\text { GT- } \\
\text { Val }\end{array}$ & $\begin{array}{c}-\mathrm{A} \\
+ \\
\end{array}$ & $\begin{array}{l}-\mathrm{G}- \\
\text { Arg }\end{array}$ & $\begin{array}{l}-\mathrm{C}- \\
\text { Thr }\end{array}$ & Del & *-- \\
\hline E2-C & T93 & $\begin{array}{l}--- \\
*\end{array}$ & $\begin{array}{c}--- \\
*\end{array}$ & $\begin{array}{l}-\mathrm{T}- \\
\text { Met }\end{array}$ & $\begin{array}{c}--\mathrm{A} \\
+\end{array}$ & --- & $\begin{array}{l}--- \\
*\end{array}$ & $\begin{array}{l}--- \\
*\end{array}$ & $\begin{array}{l}--- \\
*\end{array}$ \\
\hline
\end{tabular}




\begin{tabular}{|c|c|c|c|c|c|c|c|c|c|c|}
\hline & T94 & $\begin{array}{l}--- \\
*\end{array}$ & $\begin{array}{l}--- \\
*\end{array}$ & $\begin{array}{l}-\mathrm{T}- \\
\text { Met }\end{array}$ & $\begin{array}{c}-\mathrm{A} \\
+\end{array}$ & *-- & $\begin{array}{l}--- \\
*\end{array}$ & \multicolumn{2}{|c|}{--- } & --- \\
\hline \multirow{3}{*}{$\begin{array}{c}\text { Sub } \\
\text { types }\end{array}$} & \multirow{2}{*}{$\begin{array}{c}\text { Amino } \\
\text { Acid }\end{array}$} & 237 & 241 & \multicolumn{2}{|l|}{245} & 246 & 279 & 280 & 357 & 358 \\
\hline & & \multicolumn{9}{|c|}{ NLS } \\
\hline & WT & $\begin{array}{c}\text { GAA } \\
\text { Glu }\end{array}$ & - & $\begin{array}{c}\text { CCA } \\
\text { Pro }\end{array}$ & & $\begin{array}{c}\text { CGC } \\
\text { Arg }\end{array}$ & $\begin{array}{c}\text { CCG } \\
\text { Pro }\end{array}$ & $\begin{array}{l}\text { ACT } \\
\text { Thr }\end{array}$ & $\begin{array}{c}\text { CAA } \\
\text { Lys }\end{array}$ & $\begin{array}{c}\text { GG } \\
\text { G } \\
\text { Gly }\end{array}$ \\
\hline \multirow{6}{*}{ E2A } & T79 & --- & $\begin{array}{l}\text { CTC } \\
\text { Leu }\end{array}$ & $\begin{array}{l}-A- \\
\text { Gln }\end{array}$ & & $\begin{array}{l}--\mathrm{T} \\
\text { Ser }\end{array}$ & $\begin{array}{c}-\mathrm{A} \\
+\end{array}$ & $\begin{array}{l}-A- \\
\text { Asn }\end{array}$ & --- & --- \\
\hline & T80 & $\begin{array}{l}-\mathrm{C}- \\
\text { Ala }\end{array}$ & --- & $\begin{array}{l}-\mathrm{A}- \\
\text { Gln }\end{array}$ & & $\begin{array}{l}--\mathrm{T} \\
\text { Ser }\end{array}$ & $\begin{array}{c}-\mathrm{A} \\
+\end{array}$ & $\begin{array}{l}-A- \\
\text { Asn }\end{array}$ & $\begin{array}{l}--- \\
*\end{array}$ & $\begin{array}{c}--- \\
*\end{array}$ \\
\hline & T86 & *-- & $\begin{array}{l}\text { CTC } \\
\text { Leu }\end{array}$ & $\begin{array}{l}-A- \\
\text { Gln }\end{array}$ & & $\begin{array}{l}--\mathrm{T} \\
\text { Ser }\end{array}$ & $\begin{array}{c}-\mathrm{A} \\
+ \\
\end{array}$ & $\begin{array}{l}-A- \\
\text { Asn }\end{array}$ & --- & $\begin{array}{l}--- \\
*\end{array}$ \\
\hline & T90 & $\begin{array}{l}-\mathrm{C}- \\
\text { Ala }\end{array}$ & --- & $\begin{array}{l}-A- \\
\text { Gln }\end{array}$ & & $\begin{array}{l}--\mathrm{T} \\
\text { Ser }\end{array}$ & $\begin{array}{c}-\mathrm{A} \\
+\end{array}$ & $\begin{array}{l}-A- \\
\text { Asn }\end{array}$ & --- & *-- \\
\hline & T91 & $\begin{array}{c}--- \\
*\end{array}$ & $\begin{array}{l}\text { CTC } \\
\text { Leu }\end{array}$ & $\begin{array}{l}-A- \\
\text { Gln }\end{array}$ & & $\begin{array}{l}--\mathrm{T} \\
\text { Ser }\end{array}$ & $\begin{array}{c}-\mathrm{A} \\
+\end{array}$ & $\begin{array}{l}-A- \\
\text { Asn }\end{array}$ & $\begin{array}{l}--- \\
*\end{array}$ & $\begin{array}{l}--- \\
*\end{array}$ \\
\hline & T97 & $\begin{array}{l}-\mathrm{C}- \\
\text { Ala }\end{array}$ & --- & $\begin{array}{l}\text {-A- } \\
\text { Gln }\end{array}$ & & $\begin{array}{l}--\mathrm{T} \\
\text { Ser }\end{array}$ & $\begin{array}{c}-\mathrm{A} \\
+ \\
\end{array}$ & $\begin{array}{l}-A- \\
\text { Asn }\end{array}$ & $\begin{array}{l}--- \\
*\end{array}$ & --- \\
\hline \multirow{2}{*}{ E2-C } & T93 & $\begin{array}{l}--- \\
*\end{array}$ & $\begin{array}{l}--- \\
* \\
\end{array}$ & --- & & --- & --- & --- & Del & Del \\
\hline & T94 & --- & --- & $\begin{array}{l}--- \\
*\end{array}$ & & --- & $\begin{array}{l}--- \\
*\end{array}$ & --- & Del & Del \\
\hline
\end{tabular}

\begin{tabular}{|c|c|c|c|c|c|c|c|c|c|c|}
\hline \multirow{3}{*}{$\begin{array}{l}\text { Sub } \\
\text { types }\end{array}$} & \multirow{2}{*}{$\begin{array}{c}\text { Amino } \\
\text { Acid }\end{array}$} & 370 & 383 & 417 & 470 & 476 & 486 & 490 & 496 & 509 \\
\hline & & NLS & \multicolumn{5}{|c|}{ TAD domain } & \multicolumn{3}{|c|}{-} \\
\hline & WT & $\begin{array}{c}\text { CCT } \\
\text { Pro } \\
\end{array}$ & $\begin{array}{c}\text { CCT } \\
\text { Pro } \\
\end{array}$ & $\begin{array}{c}\text { ACG } \\
\text { Thr }\end{array}$ & $\begin{array}{l}\text { TCA } \\
\text { Ser }\end{array}$ & $\begin{array}{c}\text { GAG } \\
\text { Glu }\end{array}$ & $\begin{array}{c}\text { ATC } \\
\text { Ile }\end{array}$ & $\begin{array}{c}\text { CCC } \\
\text { Pro } \\
\end{array}$ & $\begin{array}{c}\text { ACC } \\
\text { Thr }\end{array}$ & $\begin{array}{c}\text { AAC } \\
\text { Assn }\end{array}$ \\
\hline \multirow{4}{*}{ E2-A } & T79 & $\begin{array}{c}-\mathrm{A} \\
+ \\
\end{array}$ & -- & $\begin{array}{c}--\mathrm{A} \\
+ \\
\end{array}$ & $\begin{array}{c}--\mathrm{T} \\
+ \\
\end{array}$ & $\begin{array}{l}\text {-G- } \\
\text { Gly }\end{array}$ & $\begin{array}{l}-\mathrm{C}- \\
\text { Thr }\end{array}$ & $\begin{array}{l}\text { A-- } \\
\text { Thr }\end{array}$ & $\begin{array}{l}-- \\
*\end{array}$ & $\begin{array}{l}\text { G-- } \\
\text { Asp }\end{array}$ \\
\hline & T80 & $\begin{array}{c}-\mathrm{A} \\
+ \\
\end{array}$ & $\begin{array}{l}--- \\
*\end{array}$ & $\begin{array}{c}--\mathrm{A} \\
+ \\
\end{array}$ & $\begin{array}{c}--\mathrm{T} \\
+ \\
\end{array}$ & $\begin{array}{l}\text {-G- } \\
\text { Gly }\end{array}$ & $\begin{array}{l}-\mathrm{C}- \\
\text { Thr }\end{array}$ & $\begin{array}{l}\text { A-- } \\
\text { Thr }\end{array}$ & $\begin{array}{l}-- \\
*\end{array}$ & $\begin{array}{l}\text { G-- } \\
\text { Asp }\end{array}$ \\
\hline & T86 & $\begin{array}{c}-A \\
+ \\
\end{array}$ & -- & $\begin{array}{c}-\mathrm{A} \\
+\end{array}$ & $\begin{array}{c}--T \\
+\end{array}$ & $\begin{array}{l}\text {-G- } \\
\text { Gly }\end{array}$ & $\begin{array}{l}-\mathrm{C}- \\
\text { Thr }\end{array}$ & $\begin{array}{l}\text { A-- } \\
\text { Thr }\end{array}$ & $\begin{array}{l}-- \\
*\end{array}$ & $\begin{array}{l}\text { G-- } \\
\text { Asp }\end{array}$ \\
\hline & T90 & $\begin{array}{c}--\mathrm{A} \\
+\end{array}$ & $\begin{array}{c}-A \\
+\end{array}$ & $\begin{array}{c}-A \\
+\end{array}$ & $\begin{array}{c}--T \\
+\end{array}$ & $\begin{array}{l}\text {-G- } \\
\text { Gly }\end{array}$ & $\begin{array}{l}\text {-C- } \\
\text { Thr }\end{array}$ & $\begin{array}{l}\text { A-- } \\
\text { Thr }\end{array}$ & $\begin{array}{l}-- \\
*\end{array}$ & $\begin{array}{l}\text { G-- } \\
\text { Asp }\end{array}$ \\
\hline
\end{tabular}




\begin{tabular}{|c|c|c|c|c|c|c|c|c|c|c|}
\hline & T91 & $\begin{array}{c}-\mathrm{A} \\
+\end{array}$ & $\begin{array}{l}--- \\
* \\
\end{array}$ & $\begin{array}{c}-\mathrm{A} \\
+ \\
\end{array}$ & $\begin{array}{c}--\mathrm{T} \\
+ \\
\end{array}$ & $\begin{array}{l}\text {-G- } \\
\text { Gly }\end{array}$ & $\begin{array}{l}\text {-C- } \\
\text { Thr }\end{array}$ & $\begin{array}{l}\text { A-- } \\
\text { Thr }\end{array}$ & $\begin{array}{l}-- \\
*\end{array}$ & $\begin{array}{l}\text { G-- } \\
\text { Asp }\end{array}$ \\
\hline & T97 & $\begin{array}{c}--\mathrm{A} \\
+\end{array}$ & $\begin{array}{c}-\mathrm{A} \\
+ \\
\end{array}$ & $\begin{array}{c}--\mathrm{A} \\
+\end{array}$ & $\begin{array}{c}--\mathrm{T} \\
+ \\
\end{array}$ & $\begin{array}{l}\text {-G- } \\
\text { Gly }\end{array}$ & $\begin{array}{l}\text {-C- } \\
\text { Thr }\end{array}$ & $\begin{array}{l}\text { A-- } \\
\text { Thr }\end{array}$ & $\begin{array}{c}-- \\
*\end{array}$ & $\begin{array}{l}\text { G-- } \\
\text { Asp }\end{array}$ \\
\hline \multirow{2}{*}{ E2-C } & T93 & $\begin{array}{c}-\mathrm{A} \\
+ \\
\end{array}$ & $\begin{array}{l}--- \\
*\end{array}$ & --- & --- & --- & $\begin{array}{c}--- \\
*\end{array}$ & $\begin{array}{l}--- \\
*\end{array}$ & $\begin{array}{l}\text {-A- } \\
\text { Asn }\end{array}$ & $\begin{array}{l}--- \\
*\end{array}$ \\
\hline & T94 & $\begin{array}{c}--\mathrm{A} \\
+\end{array}$ & $\begin{array}{l}--- \\
* \\
\end{array}$ & --- & --- & $\begin{array}{l}--- \\
*\end{array}$ & --- & --- & $\begin{array}{l}\text {-A- } \\
\text { Asn }\end{array}$ & $\begin{array}{l}--- \\
*\end{array}$ \\
\hline
\end{tabular}

Note: (-) a position with the same nucleotide as Wild type sequence; $\left(^{*}\right)$ a position with the same amino acid as Wild type sequence; $(+)$ a position with the same amino acid as Wild type on silent mutations, and (Del) a position with the deleted amino acid

Source: The researcher's data analysis

$E B N A-2$ is one of the first latent proteins detected after an EBV infection and primarily upregulates the expression of viral and cellular genes. As the functions of EBNA-2, it interacts with other transcription factors associated with the Notch signaling pathway (Henkel, Ling, Hayward, \& Peterson, 1994; Hsieh et al., 1996). The variants of EBNA-2 have evidently been involved in the NPC carcinoma tumorigenesis. According to previous studies on EBNA-2 gene polymorphisms in gastric carcinoma and NPCs, the variant of E-2A was significantly detected only in NPC and more important in the pathogenesis of NPC (Wang et al., 2012). As for the $E B N A-2$ protein structure, the three important domains for the transcription regulation function of EBNA-2 including the self-association domain (122-232 aa), trans-activation domain (TAD; 431-487 aa) and nuclear localization signals (NLS; 244-378 aa, and 466-483 aa) (Cohen, Wang, $\&$ Kieff, 1991) were sequenced and analyzed in this study. In detail, experimental samples of E2-A variants were divided into two distinct groups with group 1 having the characteristic of the mutation loss of $51 \mathrm{bp}$ at 49103 and group 2 containing the three-nucleotide CTC insertion mutation at 49136. The DNA pattern of E-2A group 1 subtype carried 11 amino acid changes at residue 151 (ATT:Ile $\rightarrow$ ATG:Met), 163 (AGG:Arg $\rightarrow$ GTG:Val), 186 (CAA:Gln $\rightarrow$ CGA:Arg), 237 (GAA:Glu $\rightarrow$ GCA:Ala), 245 (CCA:Pro $\rightarrow$ CAA:Gln), 246 (CGC:Arg $\rightarrow$ CGT:Ser), 280 (ACT: Thr $\rightarrow$ AAT: Asn), 476 (GAG:Glu $\rightarrow$ GGG:Gly), 486 (ATC:Ile $\rightarrow$ ACC:Thr), 490 (CCC:Pro $\rightarrow$ ACC:Thr), 509 (AAC:Asn $\rightarrow$ GAC:Asp) and one deletion at 200217 (Del), represented by T80 sequence. The second pastern of group 2, which was represented by T69, carried 11 amino acid changes at residue 151 (ATT:Ile $\rightarrow$ ATG:Met), 163 (ACG:Arg $\rightarrow$ GTG:Val), 185 (CAA:Gln $\rightarrow$ CGA:Arg), 195 (ATG:Met $\rightarrow$ ACG:Thr), 245 (CCA:Pro $\rightarrow$ CAA:Gln), 246 (CGC:Arg $\rightarrow$ CGT:Ser), 280 (ACT:Thr $\rightarrow$ AAT: Asn), 476 (GAG:Glu $\rightarrow$ GGG:Gly), 468 (ATC:Ile $\rightarrow$ ACC:Thr), 490 (CCC:Pro $\rightarrow$ ACC:Thr), 509 (AAC:Asn $\rightarrow$ GAC:Asp) and one insertion in 241 (CTC: Leu). In the current study, the variants E2-A was identified as the predominant subtype in our samples, which was also characterized as the frequent subtype in other Asian countries, including Hong Kong (Kwok et al., 2014), Southern China (Wu et al., 2015).

\section{Conclusion}

In conclusion, this study described the subtypes of EBNA-2 polymorphisms in Vietnamese NPC biopsy samples, including E-2A and E-2C. Of the EBNA-2 subtypes, E-2A was the most prevalent $E B N A-2$ subtype in Vietnamese patients, in which two different patterns 
were identified. Considering the shortcomings of this work, our finding provided the initial data for the potential contribution of EBNA-2 polymorphisms to the etiology of endemic NPC in the Vietnamese population. A larger number and direct comparison of various sample sources such as throat washing should be included in future research. It will be very useful to indicate the sample type specificity of genotyping changes to apply in genetic screening of NPC in the Vietnamese population.

\section{ACKNOWLEDGEMENTS}

We wish to express our sincere thanks to the research project sponsored by Ho Chi Minh City Open University. We are grateful to all recruited participants in this work and all staff members of Otorhinolaryngology in Cho Ray Hospital, Ho Chi Minh City for collecting the samples used in this study.

\section{References}

Cohen, J. I., Wang, F., \& Kieff, E. (1991). Epstein-Barr virus nuclear protein 2 mutations define essential domains for transformation and transactivation. Journal of Virology, 65, 25452554.

da Costa, V. G., Marques-Silva, A. C., \& Moreli, M. L. (2015). The Epstein-Barr virus latent membrane protein-1 (LMP1) 30-bp deletion and XhoI-polymorphism in nasopharyngeal carcinoma: A meta-analysis of observational studies. Systematic Reviews, 13, 4-46.

Dai, W., Zheng, H., Cheung, A. K., \& Lung, M. L. (2016). Genetic and epigenetic landscape of nasopharyngeal carcinoma. Chinese Clinical Oncology, 5(2), 16-28.

Henkel, T., Ling, P. D., Hayward, S. D., \& Peterson, M. G. (1994). Mediation of Epstein-Barr virus EBNA2 transactivation by recombination signal binding protein $\mathrm{J}$ kappa. Science, 265, 92-95.

Hsieh, J. J., Henkel, T., Salmon, P., Robey, E., Peterson, M. G., \& Hayward, S. D. (1996). Truncated mammalian Notch1 activates CBF1/RBPJk-repressed genes by a mechanism resembling that of Epstein-Barr virus EBNA2. Molecular and Cellular Biology, 16(3), 952-959.

Ko, Y. H. (2015). EBV and human cancer. Experimental \& Molecular Medicine, 47(1), e130.

Kwok, H., Wu, C. W., Palser, A. L., Kellam, P., Sham, P. C., Kwong, D. L., \& Chiang, A. K. (2014). Genomic diversity of Epstein-Barr virus genomes isolated from primary nasopharyngeal carcinoma biopsy samples. Journal of Virology, 88(18), 10662-10672.

Lo, K. W., To, K. F., \& Huang, D. P. (2004). Focus on nasopharyngeal carcinoma. Cancer Cell, $5(5), 423-428$.

McDermott, A. L., Dutt, S. N. \& Watkinson, J. C. (2001). The aetiology of nasopharyngeal carcinoma. Clinical Otolaryngology, 26(2), 82-92. 
Pathmanathan, R., Prasad, U., Sadler, R., Flynn, K., \& Raab-Traub, N. (1995). Clonal proliferations of cells infected with Epstein-Barr virus in preinvasive lesions related to nasopharyngeal carcinoma. New England Journal of Medicine, 333(11), 693-698.

Tsao, S. W., Yip, Y. L., Tsang, C. M., Pang, P. S., Lau, V. M., Zhang, G., \& Lo, K. W. (2014). Etiological factors of nasopharyngeal carcinoma. Oral Oncology, 50(5), 330-338.

Wang, X., Wang, Y., Wu, G., Chao, Y., Sun, Z., \& Luo, B. (2012). Sequence analysis of Epstein-Barr virus EBNA-2 gene coding amino acid 148-487 in nasopharyngeal and gastric carcinomas. Virology Journal, 9, 49.

Wu, G., Liu, X., Liu, S., Shu, J., Sun, Z., \& Luo, B. (2015). Epstein-Barr Virus EBNA-2 Polymorphic Patterns in Nasopharyngeal Carcinoma in Southern China. Intervirology, 58(6), 386-392.

Yang, X. R., Diehl, S., Pfeiffer, R., Chen, C. -J., Hsu, W. -L., Dosemeci, M., ... Hildesheim, A. (2005). Evaluation of risk factors for nasopharyngeal carcinoma in high-risk nasopharyngeal carcinoma families in Taiwan. Cancer Epidemiology, Biomarkers \& Prevention, 14(4), 900-905. 